\title{
Mortality after admission for acute myocardial infarction in Aboriginal and non-Aboriginal people in New South Wales, Australia: a multilevel data linkage study
}

Deborah A Randall ${ }^{1 *}$, Louisa R Jorm², ${ }^{1,}$ Sanja Lujic ${ }^{1}$, Aiden J O'Loughlin ${ }^{1}$, Timothy R Churches², Mary M Haines², Sandra J Eades ${ }^{3}$ and Alastair H Leyland ${ }^{4}$

\begin{abstract}
Background: Heart disease is a leading cause of the gap in burden of disease between Aboriginal and nonAboriginal Australians. Our study investigated short- and long-term mortality after admission for Aboriginal and non-Aboriginal people admitted with acute myocardial infarction (AMI) to public hospitals in New South Wales, Australia, and examined the impact of the hospital of admission on outcomes.
\end{abstract}

Methods: Admission records were linked to mortality records for 60047 patients aged 25-84 years admitted with a diagnosis of AMI between July 2001 and December 2008. Multilevel logistic regression was used to estimate adjusted odds ratios (AOR) for 30- and 365-day all-cause mortality.

Results: Aboriginal patients admitted with an AMI were younger than non-Aboriginal patients, and more likely to be admitted to lower volume, remote hospitals without on-site angiography. Adjusting for age, sex, year and hospital, Aboriginal patients had a similar 30-day mortality risk to non-Aboriginal patients (AOR: 1.07; 95\% Cl 0.831.37) but a higher risk of dying within 365 days (AOR: 1.34; 95\% Cl 1.10-1.63). The latter difference did not persist after adjustment for comorbid conditions (AOR: 1.12; $95 \% \mathrm{Cl}$ 0.91-1.38). Patients admitted to more remote hospitals, those with lower patient volume and those without on-site angiography had increased risk of short and long-term mortality regardless of Aboriginal status.

Conclusions: Improving access to larger hospitals and those with specialist cardiac facilities could improve outcomes following AMl for all patients. However, major efforts to boost primary and secondary prevention of AMI are required to reduce the mortality gap between Aboriginal and non-Aboriginal people.

Keywords: Hospital performance, Acute myocardial infarction, Ischaemic heart disease, Aboriginal health, Health outcomes, Multilevel modelling, Data linkage

\section{Background}

The health of Aboriginal and Torres Strait Islander Australians is worse than that of other Australians across every conceivable health indicator [1]. The determinants of the disproportionate ill-health among Aboriginal people include higher levels of behavioural, biomedical and psychosocial risk factors, in combination with lesser

\footnotetext{
* Correspondence: d.randall@uws.edu.au

'School of Medicine, University of Western Sydney, Narellan Road, Campbelltown, NSW, Australia

Full list of author information is available at the end of the article
}

access to appropriate health services and lower socioeconomic status (SES) [1-5].

While the determinants are complex, the results are clear - Aboriginal Australians have a burden of disease which is two-and-a-half times that of non-Aboriginal Australians [1], and an estimated gap in life expectancy that is greater than that in other developed countries [6]. Ischaemic heart disease (IHD) accounts for $14 \%$ of the gap in burden of disease [2], and Aboriginal Australians have higher age-adjusted rates of incidence, hospital admission and mortality for acute myocardial infarction 
(AMI), the acute form of IHD [3,7-9]. While several studies have compared rates of invasive interventions [7,9-11], none has quantified the impact of hospital care on variations in short-term and long-term outcomes for Aboriginal and non-Aboriginal people after admission for AMI.

This study investigated short- and long-term mortality after admission for Aboriginal and non-Aboriginal residents of New South Wales (NSW) admitted to hospital with AMI and also investigated the impact of hospital of admission on outcomes.

\section{Methods \\ Study design}

Observational cohort study using linked hospital and mortality data.

\section{Data sources}

The NSW Admitted Patients Data Collection (APDC) includes records for all NSW public and private hospital separations (hospital admissions ending in a discharge, transfer, type-change or death). Patient demographics and multiple diagnoses and procedures are recorded for each separation and coded according to the Australian modification of the International Statistical Classification of Diseases and Related Problems (diagnoses) and the Australian Classification of Health Interventions (procedures) [12]. The NSW Register of Births, Deaths and Marriages (RBDM) captures details of all deaths registered in NSW.

\section{Probabilistic linkage}

The APDC from 1 July 2000 to 31 December 2008 was linked with the RBDM from 1 July 2000 to 31 December 2009. Personal identifiers (including full name, date of birth, sex and address) from the datasets were linked using probabilistic methods by the Centre for Health Record Linkage [13]. The researchers were supplied with de-identified APDC and RBDM data and merged these using a project-specific unique person number.

\section{Setting}

NSW is the most populous state in Australia with an estimated 6.8 million residents in 2006, $2.2 \%$ who identify as Aboriginal and/or Torres Strait Islander [14]. Approximately $30 \%$ of Australia's Aboriginal peoples live in NSW, the largest percentage of all the States and Territories in Australia. In 2006, 73\% of the total NSW population lived in a major city [15] compared with $42 \%$ of the NSW Aboriginal population [16]. The median age of Aboriginal people in NSW in 2006 was 20.6 years [17] while the median age for non-Aboriginal people was 38.6 years [18].

\section{Participants}

The participants were NSW residents aged 25 to 84 who were admitted to a public hospital with a primary diagnosis of acute myocardial infarction (AMI, ICD-10-AM code 'I21') or ischaemic heart disease (IHD, ICD-10-AM codes 'I20'-'I25') with a diagnosis of AMI in the second or third diagnosis fields, and where the admission was classified as both 'acute care' and 'emergency'. Only first admissions to public hospitals were included, because the linkage for private hospitals was not of the same quality as for public hospitals. The first such admission in the period July 2001 to December 2008 was chosen as the index admission for analysis, with at least a one-year clearance period for previous admissions for AMI. The cohort thus consisted of cases whose index admission was their first-ever as well as those who had an AMI admission prior to July 2000. A sensitivity analysis excluding previously-admitted cases with clearance periods of between one and four years found no significant difference in the Aboriginal to non-Aboriginal 30-day and 365-day mortality ratios. Patients were excluded if they had missing data or appeared to be duplicate admissions (244 non-Aboriginal and 3 Aboriginal records). The excluded records had the same percentage of deaths within 30 days as the final data set (9\%). The final data set included 60047 patients (1183 Aboriginal, 58864 non-Aboriginal) admitted to 174 public hospitals in NSW.

\section{Analysis variables}

The main outcomes were 30-day and 365-day all-cause mortality after hospital admission. The main variable of interest was whether the patient identified as Aboriginal. This was determined based on the standard question, "Are you of Aboriginal or Torres Strait Islander origin?", recorded in the hospital data. In 2007, an audit was conducted and the percentage of Aboriginal and Torres Strait Islander patients correctly identified in NSW public hospitals was estimated to be $88 \%$ [19]. While identification is thought to have improved over time, there were no audits previously published for NSW [20]. However, the Australian Institute of Health and Welfare used an under-identification factor of $30 \%$ to correct expenditure data for 1998-99 and 2001-02 for NSW hospital data [20]. Probabilistic linkage provided opportunities for identification across the entire admission history for each individual but in the absence of an external source of Aboriginal status to validate identification algorithms, we defined Aboriginal people in our study (Aboriginal and/or Torres Strait Islander) based on the most recent public hospital admission recorded for each person. This was thought to be the most accurate method due to improvements in identification over time $[19,20]$. A sensitivity analysis was carried out using two alternative 
definitions: identified as Aboriginal on every admission ('all admissions') or on at least one admission ('ever identified').

Comorbidities were measured with the Ontario AMI mortality prediction rule (OAMIMPR) [21] conditions, developed in Ontario, Canada for risk adjustment specifically after AMI admission, and were supplemented with additional Charlson Comorbidity Index conditions [22] that had a significant age-, sex- and year-adjusted association with 30-day or 365-day mortality. All comorbidities were collected with a one-year look-back that included any comorbid conditions recorded on the APDC for each person for a full year before the AMI admission as well as on the admission record. Socio-economic status was classified using the ABS Socio-Economic Index for Areas Index of Relative Social Disadvantage (SEIFA IRSD) based on Statistical Local Area (SLA) of residence, and divided into population quintile groups. Remoteness of residence was ascertained using the Accessibility/Remoteness Index of Australia (ARIA+) for SLA of residence, grouped into four categories (major city, inner regional, outer regional and remote/very remote). The hospital of analysis was the first hospital of admission in the AMI admission episode. There were three hospital-level variables: hospital remoteness (ARIA + group of the hospital based on postcode), hospital size (the average number of all acute admissions per year between 2001 and 2008, calculated for each hospital and divided into five groups at the 50th, 75th, 85th and 95th percentiles for hospitals), and the presence or absence of on-site cardiac angiography facilities.

\section{Statistical analysis}

Characteristics of Aboriginal and non-Aboriginal people admitted with AMI were compared using $x^{2}$ tests. Comorbidities were additionally compared using age-, sex- and year-adjusted prevalence ratios calculated using a log-Poisson model. A series of multilevel logistic regression models with 60047 AMI patients clustered within 174 hospitals investigated: the relative odds of 30day and 365-day mortality after admission for Aboriginal people compared with non-Aboriginal people with stepwise adjustment of individual and hospital factors; how much of the variation in mortality related to the hospital of admission; what individual characteristics are associated with 30-day and 365-day mortality; and what hospital characteristics might explain residual variation between hospitals. The number of AMI patients per hospital in the final models ranged from 1 to 2691, with a median of 65 . Only $5 \%$ of hospitals had two or fewer patients. Multilevel modelling accounts for the clustering of patients within hospitals and also partitions the residual variation into the between-hospital variation and within-hospital variation [23]. All multilevel models had a random intercept allowing the hospital mortality rate to vary, and we also tested random slope models to see if the odds ratio for Aboriginal status varied between hospitals. The hospital-level variance can be expressed as a percentage of the total variance, also called the intraclass correlation coefficient (ICC), or can be converted into a median odds ratio (MOR), which is the median of the odds ratios of pair-wise comparisons of patients with identical characteristics taken from randomly chosen hospitals [24]. Data analyses were carried out using SAS 9.1.3 [25] and MLwiN 2.22 [26].

\section{Ethics approval}

Approval for the study was given by the NSW Population and Health Services Research Ethics Committee, the Aboriginal Health and Medical Research Council of NSW Ethics Committee, and the University of Western Sydney Human Research Ethics Committee.

\section{Results}

\section{Patient characteristics}

Aboriginal patients with AMI were significantly younger than non-Aboriginal patients with just over half of the Aboriginal patients aged 25-54 years compared with only one-fifth of non-Aboriginal patients (Table 1). Aboriginal patients were also more likely to be female, more likely to be living in an area classified as most disadvantaged, and more likely to be living in an outer regional or remote area. Aboriginal patients were significantly less likely to be admitted to a major city hospital, a hospital with 18400 or more average acute admissions per year, or one with on-site angiography facilities. Due to the marked demographic differences, age-, sex- and year-adjusted prevalence ratios were calculated to compare the prevalence of comorbidities. These showed that Aboriginal patients were more likely than non-Aboriginal patients of the same age, sex and year of admission to have acute and chronic renal failure, paraplegia, congestive heart failure, diabetes with complications, and pulmonary disease (Figure 1).

\section{Short- and long-term mortality after admission}

Of the 1183 Aboriginal patients admitted with AMI, 70 died within 30 days of admission (5.9\%) and 127 died within one year of admission (10.7\%). Of the 58864 nonAboriginal patients admitted with AMI, 5474 died within 30 days $(9.3 \%)$ and 9148 died within one year (15.2\%). When accounting only for hospital of admission through the random intercept multilevel model, Aboriginal patients with AMI had lower odds of dying within 30 days than non-Aboriginal patients (odds ratio (OR) 0.61; 95\% CI 0.48-0.78; Table 2, Model 1A). However, after adjusting for age, sex and year of admission there was no significant difference in 30-day mortality 
Table 1 Individual and hospital characteristics of Aboriginal and non-Aboriginal people admitted with acute myocardial infarction

\begin{tabular}{cccc}
\hline $\begin{array}{c}\text { Aboriginal } \\
(\mathrm{n}=1183)\end{array}$ & $\begin{array}{c}\text { Non- } \\
\text { Aboriginal } \\
(\mathrm{n}=58877)\end{array}$ & $\begin{array}{c}\mathrm{X}^{2} \\
\mathrm{p} \text {-value }\end{array}$ \\
\hline$N$ & $\%$ & $N \quad \%$
\end{tabular}

\section{Individual characteristic}

Age group

$25-34$
$35-44$
$45-54$
$55-64$
$65-74$
$75-84$
Sex

Male

Female

Comorbid conditions $^{a}$

Diabetes with

complications

Cardiac dysrhythmias

Congestive heart failure

Pulmonary disease

Chronic renal failure

Acute renal failure

Cerebrovascular

disease

Paraplegia

Cancer

Peripheral vascular disease

\section{Shock}

Pulmonary oedema

Connective tissue

disorder

Dementia

Severe liver disease

Liver disease

Socio-economic status ${ }^{b}$

$1^{\text {st }}$ quintile - least

disadvantaged

$2^{\text {nd }}$ quintile

$3^{\text {rd }}$ quintile

$4^{\text {th }}$ quintile

$5^{\text {th }}$ quintile - most

disadvantaged

$\begin{array}{rrrrr}47 & 4.0 & 421 & 0.7 & <.001 \\ 257 & 21.7 & 2829 & 4.8 & \\ 360 & 30.4 & 8579 & 14.6 & \\ 265 & 22.4 & 13144 & 22.3 & \\ 180 & 15.2 & 15410 & 26.2 & \\ 74 & 6.3 & 18481 & 31.4 & \end{array}$

$\begin{array}{lllll}727 & 61.5 & 39950 & 67.9 & <.001 \\ 456 & 38.5 & 18914 & 32.1 & \end{array}$

$\begin{array}{lllll}279 & 23.6 & 8903 & 15.1 & <.001\end{array}$

$\begin{array}{llllll}185 & 15.6 & 12539 & 21.3 & <.001\end{array}$

$\begin{array}{lllll}154 & 13.0 & 8350 & 14.2 & .254\end{array}$

$\begin{array}{lllll}136 & 11.5 & 5236 & 8.9 & .002\end{array}$

$\begin{array}{lllll}96 & 8.1 & 4143 & 7.0 & .152\end{array}$

$\begin{array}{lllll}47 & 4.0 & 2729 & 4.6 & .282\end{array}$

$\begin{array}{lllll}36 & 3.0 & 2552 & 4.3 & .030\end{array}$

$\begin{array}{lllll}25 & 2.1 & 1341 & 2.3 & .707\end{array}$

$\begin{array}{lllll}12 & 1.0 & 1108 & 1.9 & .029\end{array}$

$\begin{array}{lllll}11 & 0.9 & 1195 & 2.0 & .008\end{array}$

$\begin{array}{lllll}9 & 0.8 & 685 & 1.2 & .199\end{array}$

$\begin{array}{lllll}8 & 0.7 & 743 & 1.3 & .073\end{array}$

$\begin{array}{lllll}5 & 0.4 & 506 & 0.9 & .105\end{array}$

$\begin{array}{lllll}2 & 0.2 & 103 & 0.2 & .962\end{array}$

$\begin{array}{lllll}1 & 0.1 & 113 & 0.2 & .615\end{array}$

$\begin{array}{lllll}23 & 1.9 & 7832 & 13.3 & <.001\end{array}$

$\begin{array}{llll}109 & 9.2 & 9946 & 16.9\end{array}$

$\begin{array}{llll}186 & 15.7 & 12726 & 21.6\end{array}$

$\begin{array}{llll}299 & 25.3 & 13102 & 22.3\end{array}$

$\begin{array}{llll}566 & 47.8 & 15258 & 25.9\end{array}$ $\begin{array}{lllll}15 & 1.3 & 1773 & 3.0 & 0.01\end{array}$
Table 1 Individual and hospital characteristics of Aboriginal and non-Aboriginal people admitted with acute myocardial infarction (Continued)

\begin{tabular}{|c|c|c|c|c|c|}
\hline \multicolumn{6}{|l|}{$\overline{\text { Remoteness of residence }^{c}}$} \\
\hline Major city & 307 & 26.0 & 34695 & 58.9 & $<.001$ \\
\hline Inner regional & 359 & 30.3 & 16319 & 27.7 & \\
\hline Outer regional & 366 & 30.9 & 7300 & 12.4 & \\
\hline Remote/very remote & 151 & 12.8 & 550 & 0.9 & \\
\hline \multicolumn{6}{|l|}{ Hospital characteristic } \\
\hline \multicolumn{6}{|l|}{ Remoteness of hospital $^{c}$} \\
\hline Major city & 389 & 32.9 & 39456 & 67.0 & $<.001$ \\
\hline Inner regional & 247 & 20.9 & 11384 & 19.3 & \\
\hline Outer regional & 414 & 35.0 & 7297 & 12.4 & \\
\hline Remote/very remote & 133 & 11.2 & 726 & 1.2 & \\
\hline \multicolumn{6}{|c|}{ Average acute admissions per year } \\
\hline Less than 1200 & 88 & 7.4 & 1245 & 2.1 & $<.001$ \\
\hline 1200-3899 & 182 & 15.4 & 3730 & 6.3 & \\
\hline $3900-7084$ & 138 & 11.7 & 3842 & 6.5 & \\
\hline 7085-18399 & 443 & 37.4 & 19977 & 33.9 & \\
\hline 18400 or more & 332 & 28.1 & 30070 & 51.1 & \\
\hline \multicolumn{6}{|l|}{ On-site angiography } \\
\hline Yes & 315 & 26.6 & 25694 & 43.6 & $<.001$ \\
\hline No & 868 & 73.4 & 33170 & 56.4 & \\
\hline
\end{tabular}

${ }^{a}$ Comorbid conditions with one-year look-back, including comorbidities on current admission and any admissions in the previous year.

${ }^{b}$ Socio-Economic Indices for Areas (SEIFA) Index of Relative Socio-Economic Disadvantage population quintiles based on statistical local area of residence. c Accessibility/Remoteness Index of Australia (ARIA+) based on statistical local area of residence for individuals or hospital postcode for hospitals.

between Aboriginal and non-Aboriginal AMI patients (AOR 1.07; 95\% CI 0.83-1.37; Model 2A). Accounting for comorbidities, remoteness of residence, and socioeconomic status (Model 5A) reduced the adjusted odds ratio to 0.95 (0.73-1.23), indicating no significant difference in 30-day mortality. A random slope effect for Aboriginal status was tested, but there was no significant variation in the Aboriginal to non-Aboriginal 30-day mortality ratio across hospitals.

The unadjusted results for 365-day mortality were similar to the 30-day model: Aboriginal patients were less likely to die within 365 days of admission than nonAboriginal patients admitted to the same hospital (OR 0.64; 95\% CI 0.53-0.77; Table 2, Model 1B). However, after adjusting for age, sex, and year of admission, Aboriginal patients had significantly higher odds of dying within 365 days than non-Aboriginal patients admitted to the same hospital (AOR 1.34, 95\% CI 1.10-1.63; Model 2B). Again, there was no random slope effect for Aboriginal status in this model. After comorbidities were accounted for there was no longer a significant 


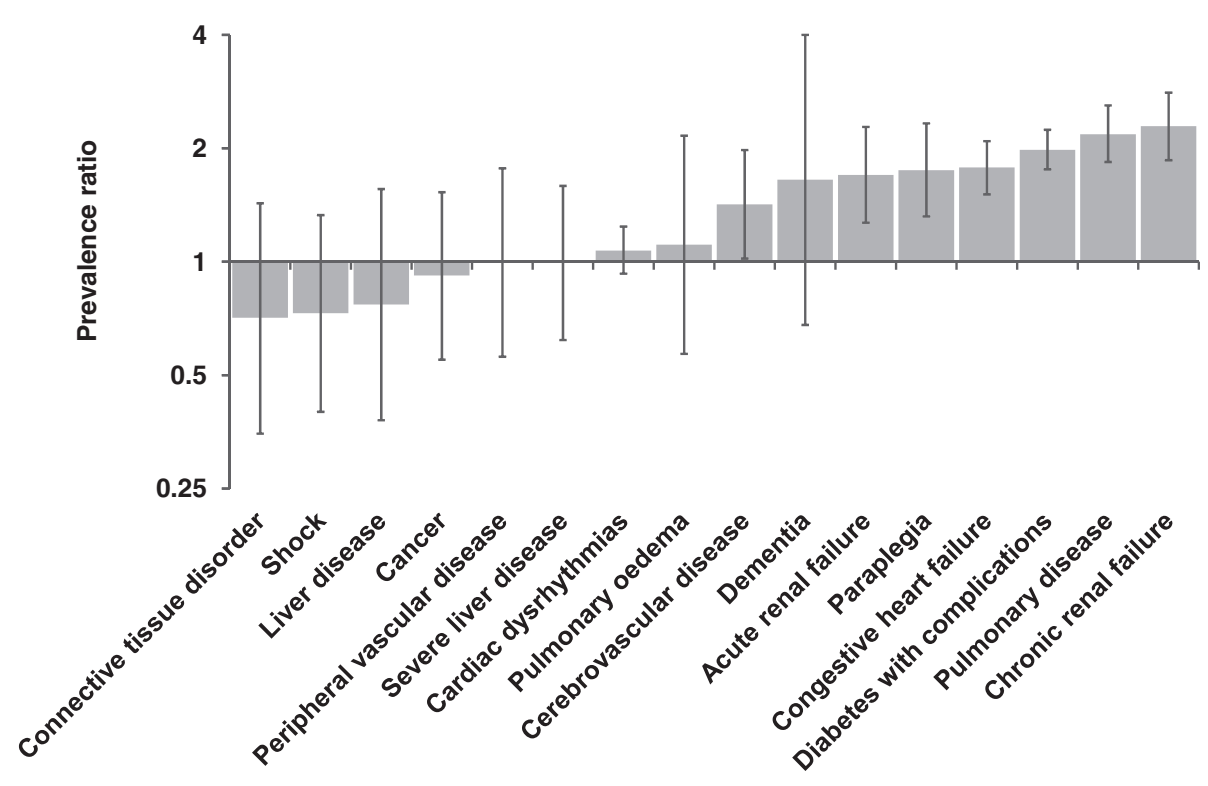

Figure 1 Relative adjusted prevalence of comorbidities for Aboriginal compared with non-Aboriginal people with AMI.Prevalence ratio adjusted for age, sex and year of admission, for Aboriginal compared with non-Aboriginal people with AMI. A value over 1 indicates that Aboriginal people have a higher prevalence of the condition, and a value under 1 indicates that non-Aboriginal people have a higher prevalence. Comorbid conditions calculated with one-year look-back, including comorbidities on current admission and any admissions in the previous year.

difference between Aboriginal and non-Aboriginal 365day mortality (AOR 1.12, 95\% CI 0.91-1.38; Model 3B).

In the fully-adjusted individual-level model for 30-day mortality (Model 5A), the percentage of unexplained variation due to the hospital of admission (or the Intraclass correlation coefficient) was $2.72 \%$. This can be expressed as a median odds ratio (MOR) of 1.34. In the fully-adjusted 365-day mortality model (Model 5B), the hospital of admission accounted for $2.58 \%$ of the unexplained variation in the outcome (MOR 1.33).

Table 3 shows odds ratios for selected individual covariates from the fully-adjusted individual-level models (Models 5A and 5B). There were no significant differences in 30-day or 365-day mortality between males and females. Older age was strongly related to both 30-day and 365-day mortality. Area of residence was not a significant predictor of 30-day or 365-day mortality, but remoteness was already being largely accounted for by adjusting for hospital of admission. Living in an area classified as the most disadvantaged was associated with higher 30-day mortality, and there was higher 365-day mortality in all the more disadvantaged quintiles compared with the least disadvantaged group. Of the comorbidities included in the model, shock was most strongly related to risk of morality, particularly 30-day mortality. Severe liver disease, cardiac dysrhythmias, dementia, cancer and acute renal failure were associated with at least a doubling in the odds of dying within 30 and 365 days of admission. Most of the other comorbidities were significantly associated with an increased risk of either 30-day or 365-day mortality. Diabetes with complications was related to a slightly lower risk of 30day mortality but a slightly higher risk of 365-day mortality.

Table 4 shows the relative odds of 30-day and 365-day mortality for the hospital characteristics, added one at a time to the fully adjusted individual-level model. Hospital remoteness was a significant predictor of both 30-day and 365-day mortality; those patients admitted to an outer regional or a remote hospital had significantly higher odds of mortality than those admitted to a major city hospital. Those admitted to hospitals with 7084 or less acute patients per year had higher odds of both 30-day and 365day mortality than those admitted to hospitals with higher numbers of acute admissions per year and there was a significant trend across groups $(\mathrm{P}<0.001$ for both 30 -day and 365-day models). Those admitted to a hospital with on-site angiography had lower odds of 30-day and 365day mortality than those admitted to a hospital without these facilities. When all three hospital level variables were added they accounted for $37 \%$ of the hospital level variation in both the 30-day and 365-day mortality models.

\section{Sensitivity analysis}

The two alternative classifications for Aboriginal status, 'ever identified' and 'all admissions', identified 1479 
Table 2 Relative odds of 30-day and 365-day mortality for Aboriginal compared with non-Aboriginal people with stepwise adjustment for covariates

\begin{tabular}{|c|c|c|c|c|}
\hline \multicolumn{5}{|c|}{ 30-day mortality models } \\
\hline Model & Adjusted for: & OR & $95 \% \mathrm{Cl}$ & $\overline{\text { p-value }}$ \\
\hline \multirow[t]{2}{*}{$1 \mathrm{~A}$} & Hospital of admission ${ }^{a}$ & 0.61 & $0.48-0.78$ & $<.001$ \\
\hline & Individual covariates & & & \\
\hline $2 \mathrm{~A}$ & + Age group, sex, year of admission & 1.07 & $0.83-1.37$ & .612 \\
\hline $3 \mathrm{~A}$ & + Comorbid conditions $^{\mathrm{b}}$ & 0.98 & $0.76-1.27$ & .886 \\
\hline $4 \mathrm{~A}$ & + Remoteness of residence $^{c}$ & 0.95 & $0.73-1.24$ & .728 \\
\hline \multirow[t]{2}{*}{$5 \mathrm{~A}$} & + Socio-economic status $^{\mathrm{d}}$ & 0.95 & $0.73-1.23$ & .684 \\
\hline & Hospital covariates $^{e}$ & & & \\
\hline $6 \mathrm{~A}$ & + Remoteness of hospital $^{\mathrm{c}}$ & 0.94 & $0.72-1.22$ & .617 \\
\hline $7 \mathrm{~A}$ & + Average acute admissions per year (- Remoteness of hospital) & 0.95 & $0.73-1.23$ & .676 \\
\hline $8 \mathrm{~A}$ & + On-site angiography (- Average acute admissions per year) & 0.94 & $0.73-1.23$ & .665 \\
\hline \multicolumn{5}{|c|}{ 365-day mortality models } \\
\hline Model & Adjusted for: & OR & $95 \% \mathrm{Cl}$ & p-value \\
\hline \multirow[t]{2}{*}{$1 \mathrm{~B}$} & Hospital of admission ${ }^{a}$ & 0.64 & $0.53-0.77$ & $<.001$ \\
\hline & Individual covariates & & & \\
\hline $2 \mathrm{~B}$ & + Age group, sex, year of admission & 1.34 & $1.10-1.63$ & .003 \\
\hline 3B & + Comorbid conditions $^{\mathrm{b}}$ & 1.12 & $0.91-1.38$ & .282 \\
\hline $4 \mathrm{~B}$ & + Remoteness of residence ${ }^{c}$ & 1.11 & $0.90-1.37$ & .317 \\
\hline \multirow[t]{2}{*}{$5 B$} & + Socio-economic status $^{\mathrm{d}}$ & 1.11 & $0.90-1.36$ & .345 \\
\hline & Hospital covariates ${ }^{e}$ & & & \\
\hline $6 \mathrm{~B}$ & + Remoteness of hospital $^{\complement}$ & 1.09 & $0.89-1.35$ & .401 \\
\hline $7 \mathrm{~B}$ & + Average acute admissions per year (- Remoteness of hospital) & 1.11 & $0.90-1.36$ & .336 \\
\hline $8 \mathrm{~B}$ & + On-site angiography (- Average acute admissions per year) & 1.10 & $0.90-1.36$ & .350 \\
\hline
\end{tabular}

$\mathrm{OR}$, odds ratio; $\mathrm{Cl}$, confidence interval.

${ }^{a}$ Hospital of admission adjusted for in a two-level random intercept model with patients nested within hospitals.

${ }^{b}$ Comorbid conditions with one-year look-back, including comorbidities on current admission and any admissions in the previous year.

c Accessibility/Remoteness Index of Australia (ARIA+) based on statistical local area of residence for individuals or hospital postcode for hospitals.

d Socio-Economic Indices for Areas (SEIFA) Index of Relative Socio-Economic Disadvantage population quintiles based on statistical local area of residence.

e Hospital covariates added one at a time to the adjusted models.

(2.5\%) and 631 (1.1\%) AMI patients as Aboriginal, respectively, compared with the 'most recent' which identified $1183(2.0 \%)$ of patients as Aboriginal. When entered into the fully-adjusted individual-level models, the 'ever identified' definition produced similar results to the 'most recent' definition, but the 'all admissions' definition resulted in higher odds of both 30-day and 365day mortality for Aboriginal compared with non-Aboriginal patients (Table 5).

\section{Discussion}

Our study is, to the best of our knowledge, the first to investigate disparities in mortality outcomes between Aboriginal and non-Aboriginal people after admission for AMI in NSW, home to $30 \%$ of Australia's Aboriginal population [14]. The overall population size and the large number of Aboriginal people residing in NSW made it possible to use multilevel modelling to examine mortality outcomes, and it is the first study of AMI hospital outcomes nationally to account for clustering of patients within hospitals and to quantify the contribution of the admitting hospital to variation in mortality outcomes.

Aboriginal and non-Aboriginal people with AMI admitted to NSW hospitals were very different. Aboriginal patients were younger, more likely to live outside of major centres and in disadvantaged areas, and more likely to be admitted to lower volume hospitals outside major centres and those without on-site angiography facilities. After adjusting for age, sex and year, they were more likely to present with comorbid conditions, including acute and chronic renal failure, diabetes, congestive heart failure and pulmonary disease. Aboriginal people in Australia have a younger age distribution than nonAboriginal people, so it is not unexpected that 
Table 3 Adjusted odds ratios for selected individual covariates for 30-day and 365-day mortality multilevel models

\begin{tabular}{|c|c|c|c|c|c|c|}
\hline & & 30-day mortz & & & 5-day mor & \\
\hline & AOR & $95 \% \mathrm{Cl}$ & p-value & AOR & $95 \% \mathrm{Cl}$ & p-value \\
\hline \multicolumn{7}{|l|}{ Sex } \\
\hline Male (ref) & 1.00 & & .211 & 1.00 & & .853 \\
\hline Female & 1.04 & $0.98-1.11$ & & 1.01 & $0.95-1.06$ & \\
\hline \multicolumn{7}{|l|}{ Age group } \\
\hline $25-34$ & 1.20 & $0.79-1.83$ & $<.001$ & 0.92 & $0.61-1.37$ & $<.001$ \\
\hline $35-44$ & 0.73 & $0.59-0.91$ & & 0.58 & $0.48-0.72$ & \\
\hline $45-54$ & 0.80 & $0.70-0.92$ & & 0.74 & $0.66-0.83$ & \\
\hline $55-64$ (ref) & 1.00 & & & 1.00 & & \\
\hline $65-74$ & 1.52 & $1.38-1.68$ & & 1.70 & $1.57-1.85$ & \\
\hline $75-84$ & 2.30 & $2.10-2.53$ & & 3.09 & $2.86-3.34$ & \\
\hline \multicolumn{7}{|l|}{ Comorbid conditions $^{\mathrm{a}}$} \\
\hline Shock & 11.54 & $10.12-13.16$ & $<.001$ & 7.94 & $6.94-9.09$ & $<.001$ \\
\hline Severe liver disease & 3.80 & $2.36-6.11$ & $<.001$ & 2.60 & $1.63-4.17$ & $<.001$ \\
\hline Cardiac dysrhythmias & 2.69 & $2.53-2.86$ & $<.001$ & 2.18 & $2.06-2.30$ & $<.001$ \\
\hline Dementia & 2.30 & $1.87-2.84$ & $<.001$ & 2.72 & $2.25-3.30$ & $<.001$ \\
\hline Cancer & 2.18 & $1.92-2.47$ & $<.001$ & 4.45 & $4.00-4.95$ & $<.001$ \\
\hline Acute renal failure & 2.02 & $1.82-2.25$ & $<.001$ & 2.00 & $1.82-2.19$ & $<.001$ \\
\hline Cerebrovascular disease & 1.53 & $1.33-1.77$ & $<.001$ & 1.47 & $1.29-1.66$ & $<.001$ \\
\hline Peripheral vascular disease & 1.32 & $1.11-1.57$ & $<.001$ & 1.30 & $1.12-1.51$ & $<.001$ \\
\hline Pulmonary disease & 1.25 & $1.14-1.37$ & $<.001$ & 1.56 & $1.45-1.67$ & $<.001$ \\
\hline Pulmonary oedema & 1.22 & $0.99-1.51$ & .056 & 1.50 & $1.26-1.80$ & $<.001$ \\
\hline Congestive heart failure & 1.20 & $1.12-1.30$ & $<.001$ & 1.81 & $1.70-1.92$ & $<.001$ \\
\hline Paraplegia & 1.08 & $0.89-1.31$ & .424 & 1.43 & $1.21-1.69$ & $<.001$ \\
\hline Chronic renal failure & 1.06 & $0.95-1.17$ & .282 & 1.56 & $1.44-1.70$ & $<.001$ \\
\hline Liver disease & 0.97 & $0.55-1.69$ & .913 & 2.42 & $1.55-3.79$ & $<.001$ \\
\hline Diabetes with complications & 0.89 & $0.82-0.97$ & .007 & 1.10 & $1.03-1.18$ & .003 \\
\hline Connective tissue disorder & 0.81 & $0.62-1.04$ & .096 & 1.18 & $0.97-1.43$ & .090 \\
\hline \multicolumn{7}{|l|}{ Remoteness of residence ${ }^{b}$} \\
\hline Major city (ref) & 1.00 & & .223 & 1.00 & & .685 \\
\hline Inner regional & 0.95 & $0.86-1.06$ & & 0.95 & $0.87-1.04$ & \\
\hline Outer regional & 1.08 & $0.94-1.24$ & & 1.00 & $0.89-1.12$ & \\
\hline Remote/very remote & 1.16 & $0.84-1.58$ & & 0.96 & $0.73-1.27$ & \\
\hline \multicolumn{7}{|l|}{ Socio-economic status ${ }^{c}$} \\
\hline $1^{\text {st }}$ quintile - least disadvantaged (ref) & 1.00 & & .033 & 1.00 & & $<.001$ \\
\hline $2^{\text {nd }}$ quintile & 1.15 & $0.99-1.32$ & & 1.11 & $0.98-1.25$ & \\
\hline $3^{\text {rd }}$ quintile & 1.10 & $0.95-1.28$ & & 1.18 & $1.04-1.34$ & \\
\hline $4^{\text {th }}$ quintile & 1.17 & $1.00-1.36$ & & 1.23 & $1.08-1.39$ & \\
\hline $5^{\text {th }}$ quintile - most disadvantaged & 1.27 & $1.08-1.48$ & & 1.32 & $1.16-1.51$ & \\
\hline
\end{tabular}

Adjusted odds ratios for selected covariates from Model 5A for 30-day mortality and Model 5B for 365-day mortality.

AOR, adjusted odds ratio; $\mathrm{Cl}$, confidence interval; Ref, referent group in the analysis.

${ }^{a}$ Comorbid conditions with one-year look-back, including comorbidities on current admission and any admissions in the previous year.

${ }^{\mathrm{b}}$ Accessibility/Remoteness Index of Australia (ARIA+) based on statistical local area of residence.

' Socio-Economic Indices for Areas (SEIFA) Index of Relative Socio-Economic Disadvantage population quintiles based on statistical local area of residence. 
Table 4 Adjusted odds ratios for selected hospital covariates for 30-day and 365-day mortality multilevel models

\begin{tabular}{|c|c|c|c|c|c|c|}
\hline & \multicolumn{3}{|c|}{ 30-day mortality } & \multicolumn{3}{|c|}{ 365-day mortality } \\
\hline & AOR & $95 \% \mathrm{Cl}$ & p-value & AOR & $95 \% \mathrm{Cl}$ & p-value \\
\hline \multicolumn{7}{|c|}{ Added into adjusted ${ }^{a}$ model separately } \\
\hline \multicolumn{7}{|l|}{ Remoteness of hospital $^{\mathrm{b}}$} \\
\hline Major city (ref) & 1.00 & & $<.001$ & 1.00 & & $<.001$ \\
\hline Inner regional & 1.15 & $0.94-1.41$ & & 1.16 & $0.97-1.39$ & \\
\hline Outer regional & 1.56 & $1.26-1.94$ & & 1.54 & $1.27-1.87$ & \\
\hline Remote/very remote & 1.83 & $1.19-2.81$ & & 1.79 & $1.22-2.61$ & \\
\hline \multicolumn{7}{|c|}{ Average acute admissions per year } \\
\hline Less than 1200 & 2.03 & $1.57-2.62$ & $<.001$ & 1.98 & $1.58-2.49$ & $<.001$ \\
\hline 1200-3899 & 1.72 & $1.39-2.13$ & & 1.55 & $1.28-1.88$ & \\
\hline $3900-7084$ & 1.36 & $1.08-1.70$ & & 1.32 & $1.07-1.62$ & \\
\hline 7085-18399 & 1.14 & $0.96-1.35$ & & 1.14 & $0.97-1.34$ & \\
\hline 18400 or more (ref) & 1.00 & & & 1.00 & & \\
\hline \multicolumn{7}{|l|}{ On-site angiography } \\
\hline Yes & 0.74 & $0.64-0.86$ & $<.001$ & 0.72 & $0.63-0.83$ & $<.001$ \\
\hline No (ref) & 1.00 & & & 1.00 & & \\
\hline
\end{tabular}

$\mathrm{AOR}$, adjusted odds ratios; $\mathrm{Cl}$, confidence interval. Ref, referent group in the analysis.

${ }^{a}$ Adjusted for Aboriginal status, age, sex, year of admission, comorbidities, remoteness of residence, socio-economic status, and a random hospital intercept, with hospital covariates added in one at a time to the model.

${ }^{\mathrm{b}}$ Accessibility/Remoteness Index of Australia (ARIA+) based on hospital postcode.

Aboriginal people admitted with AMI would be younger; however, higher age-specific incidence of AMI particularly among younger Aboriginal people was recently reported by a study in Western Australia (WA) [8]. These findings and ours point to the importance of targeting the early onset of AMI among Aboriginal people and preventing or managing chronic diseases that may complicate treatment or lead to poorer long-term outcomes.

Our study found that once admitted to hospital, Aboriginal patients with AMI were less likely to die within 30 days than non-Aboriginal patients admitted to the same hospital (Table 2, Model 1A). However, this finding was explained by substantial age differences: after adjusting for age, sex and year of admission, the differences in 30-day mortality was no longer significant (Model 2A). In contrast, after adjusting for age, sex and year, Aboriginal patients had $34 \%$ higher odds of dying within one year compared with non-Aboriginal patients admitted to the same hospital (Model 2B). However, this difference was no longer significant after adjusting for selected comorbidities (Model 3B), suggesting that part of the higher one-year mortality is due to the higher comorbidity burden among Aboriginal people admitted with AMI.

Our findings regarding short-term mortality differed from those of the WA study, which reported higher postadmission 28-day mortality ratios for Aboriginal compared with non-Aboriginal patients, ranging from 1.7 in 55-
74 year-old males and females to 3.6 in $25-54$ year old males [8]. This discrepancy might relate to the different profile of the WA Aboriginal population ( $41 \%$ resident in remote or very remote areas, compared with $5 \%$ in NSW) [18], and differences in study methodology (the WA study did not account for hospital of admission).

For longer-term mortality, our findings were similar to those of a Queensland study that reported an ageadjusted risk ratio of 1.8 (95\% CI, 1.5-2.2) for 365-day mortality in Aboriginal patients with AMI after admission to Queensland public hospitals [10]. We found that the significantly higher one-year mortality for Aboriginal patients did not persist after adjusting for comorbidities, but a recent study in WA found significantly higher rates of two-year cardiovascular death or recurrent AMI for Aboriginal compared with non-Aboriginal males and females after adjusting for demographic characteristics and comorbidities [27]. These findings may suggest that the Aboriginal to non-Aboriginal disparity in mortality is greater in WA than in NSW. However, it is difficult to compare these findings directly because our study had a shorter length of follow-up for all-cause mortality, adjusted for hospital of admission, and did not examine mortality and recurrent AMI as a combined outcome. An increase in the Aboriginal to non-Aboriginal mortality ratio with increasing time after discharge has been shown in the Northern Territory for those admitted with acute coronary syndrome and surviving to discharge, 
Table 5 Relative odds of 30-day and 365-day mortality by different algorithms for identifying Aboriginal people in the hospital data

\begin{tabular}{|c|c|c|c|c|c|c|}
\hline & \multicolumn{3}{|c|}{ 30-day mortality } & \multicolumn{3}{|c|}{ 365-day mortality } \\
\hline & AOR & $(95 \% \mathrm{Cl})$ & p-value & AOR & $(95 \% \mathrm{Cl})$ & p-value \\
\hline \multicolumn{7}{|l|}{ Most recent ${ }^{a}$} \\
\hline Non-Aboriginal (ref) & 1.00 & & .684 & 1.00 & & .345 \\
\hline Aboriginal & 0.95 & $0.73-1.23$ & & 1.11 & $0.90-1.36$ & \\
\hline \multicolumn{7}{|l|}{ Ever identified ${ }^{\mathrm{b}}$} \\
\hline Non-Aboriginal (ref) & 1.00 & & .582 & 1.00 & & .510 \\
\hline Aboriginal & 0.94 & $0.74-1.18$ & & 1.06 & $0.88-1.28$ & \\
\hline \multicolumn{7}{|l|}{ All admissions ${ }^{c}$} \\
\hline Non-Aboriginal (ref) & 1.00 & & .005 & 1.00 & & $<.001$ \\
\hline Aboriginal & 1.55 & $1.14-2.10$ & & 1.61 & $1.25-2.07$ & \\
\hline
\end{tabular}

AOR, adjusted odds ratios, adjusting for age, sex, year of admission, comorbidities, remoteness of residence, socio-economic status.

$\mathrm{Cl}$, confidence interval. Ref, referent group in the analysis.

${ }^{a}$ Identified as Aboriginal in their most recent public hospital admission.

${ }^{b}$ Identified as Aboriginal in at least one public hospital admission.

c Identified as Aboriginal on all public hospital admissions.

with the disparities in mortality appearing at six months and Aboriginal patients being about three times more likely to die than non-Aboriginal patients after four years [28]. However, caution must be taken when comparing Aboriginal peoples across Australia due to the differences in culture, geographic distribution, and access to and provision of services.

Our study showed that differences between hospitals impacted on mortality outcomes for both Aboriginal and non-Aboriginal patients. After adjustment for patient factors, $2.72 \%$ of the remaining variation in 30 -day mortality was attributable to differences between hospitals. This equates to a median odds ratio of 1.34 , indicating a median difference of $34 \%$ in the odds of dying between randomly chosen pairs of hospitals. Almost $40 \%$ of this hospital-level contribution to variation in mortality was explained by hospital remoteness, hospital size and cardiac facilities. Patients admitted to smaller hospitals, and those in outer regional and remote areas, had a higher risk of short-term mortality, while patients admitted to a hospital with on-site angiography facilities had a reduced risk of dying. Recently, in the United States, condition-specific hospital volume was shown to be related to 30-day postadmission mortality after AMI, up to a threshold value, which was lower for hospitals with cardiac revascularisation services (432 vs 586 AMI admissions/year) [29]. A Canadian study also found that admission to hospitals with on-site revascularisation facilities was related to improved long-term outcomes after AMI [30]. However, our findings regarding the specific impact of hospital size, remoteness and on-site angiography facilities on outcomes should be interpreted with caution, as these variables may be correlated with other unmeasured aspects of hospital quality of care. We found no variation in the Aboriginal to non-Aboriginal mortality ratio (both short- and longterm) across hospitals.

There were limitations to our study due to using administrative data not collected for research purposes. Firstly, there was limited clinical information in the hospital data for risk adjustment; however, we used the conditions adjusted for in the Ontario AMI Mortality Risk Prediction Rule developed in Canada for use with AMI and administrative hospital data [21] and supplemented this with additional conditions from the Charlson Comorbidity Index [22]. Secondly, we were not able to remove all prevalent cases from our study because there were only a total of eight and a half years of linked data available. We did, however, test various clearance periods of up to four years and found that the Aboriginal to non-Aboriginal age-and sex-adjusted mortality ratios did not appear sensitive to the length of the clearance period. Thirdly, our sensitivity analysis using different algorithms for identifying Aboriginal people highlighted the potential for apparent disparities to be influenced by how Aboriginal status is defined. The strict definition requiring patients to be identified as Aboriginal at every hospital admission identified only $1 \%$ of admissions as Aboriginal which is half as many as the 'most recent' algorithm but generated higher relative odds of Aboriginal mortality. This may be because those people consistently identified as Aboriginal in the APDC have poorer health than Aboriginal people not consistently identified, but it may also be because the definition included a greater proportion with only a single admission, possibly skewing the sample towards people who died post-AMI. Lastly, we did not include deaths from AMI that 
occurred before the patient was admitted to hospital, either sudden death or death in ambulance or Emergency Department. It is possible that Aboriginal people would be overrepresented in these early deaths from AMI, due to higher comorbidity rates or living a greater distance from the nearest hospital, but this was outside the scope of our study examining outcomes after hospital admission.

Our study and others point to the importance of prevention and early intervention to target the early onset of AMI among Aboriginal Australians. These efforts must target risk factor prevalence among Aboriginal people, including higher rates of smoking and overweight and obesity, and the earlier onset of comorbidities like diabetes and renal failure [1]. However, poor health behaviours may be a way of coping for people living under chronically stressful conditions, so psychosocial and emotional factors must also be taken into account $[31,32]$. Importantly, our study has demonstrated that there are gains to be made-both for Aboriginal and non-Aboriginal people-by improving access to larger hospitals and hospitals with on-site angiography or by improving the cardiac care facilities at smaller hospitals.

The population density and geographic distances in Australia pose difficult policy questions about whether it is best to transfer patients as quickly as possible to major city hospitals or whether it is efficient to increase services in less densely population areas. Our results showed that the difference in outcomes for inner regional compared with major city hospitals was small and not significant, so boosting resources in regional centres may reduce the difference altogether, and reduce travel times to cardiac facilities for those living in regional and remote areas. One challenge is to ensure that any interventions are culturally appropriate for Aboriginal patients. While transfers can be very stressful for Aboriginal people living in remote areas, an action research study concluded that small interventions such as having dedicated liaison officers in the health system could improve cultural awareness of practitioners as well as communication and continuity of care and improve outcomes for Aboriginal patients [33].

The higher mortality among Aboriginal patients in the first year after admission also highlights the importance of improved post-AMI care including appropriate medication and lifestyle interventions. This period after discharge warrants further investigation to disentangle the impacts on mortality of comorbidity burden and differences in access to, or adherence with, follow-up care and secondary prevention.

\section{Conclusions}

Improving access to larger hospitals or those with specialist treatment facilities could improve outcomes following
AMI for residents of rural and regional areas, both Aboriginal and non-Aboriginal. However, major efforts to boost primary and secondary prevention of AMI are required to reduce the mortality gap between Aboriginal and nonAboriginal people.

\section{Competing interests}

The authors declare that they have no competing interest.

\section{Authors' contributions}

DR had overall responsibility for the design of this study, data management, statistical analysis and drafting this paper. $\mathrm{LJ}$ initiated the IHOPE project and provided overall oversight. LJ, SL, TC, MH, SE and AL contributed to the conception and design of the IHOPE project. SL provided advice on data management and statistical analysis. AO provided advice on clinical aspects and on hospital levels of service. AL provided oversight and advice for the design and interpretation of the statistical analyses. All authors contributed to the interpretation of findings, the writing of the paper and approved the final draft.

\section{Acknowledgements}

We would like to acknowledge the NSW Ministry of Health and NSW Register of Births, Deaths and Marriages for allowing access to the data, and the Centre for Health Record Linkage for conducting the probabilistic linkage of records. We would also like to thank the reviewers for their thoughtful comments on earlier drafts of this paper. The Indigenous Health Outcomes Patient Evaluation (IHOPE) study is funded by a National Health and Medical Research Council Project Grant (\#573113). The funding body had no role in the research project.

\section{Author details}

${ }^{1}$ School of Medicine, University of Western Sydney, Narellan Road, Campbelltown, NSW, Australia. ${ }^{2}$ The Sax Institute, Quay Street, Sydney, NSW, Australia. ${ }^{3}$ Baker IDI Heart and Diabetes Institute, Commercial Road, Melbourne, Victoria, Australia. ${ }^{4}$ Medical Research Council/Chief Scientist Office Social and Public Health Sciences Unit, Lilybank Gardens, Glasgow, UK.

Received: 17 October 2011 Accepted: 3 April 2012

Published: 10 April 2012

\section{References}

1. Australian Institute of Health and Welfare, Australian Bureau of Statistics: The Health and Welfare of Australia's Aboriginal and Torres Strait Islander Peoples, 2008. Canberra, Australia: Australian Institute of Health and Welfare and Australian Bureau of Statistics; 2008.

2. Vos T, Barker B, Begg S, Stanley L, Lopez AD: Burden of disease and injury in Aboriginal and Torres Strait Islander Peoples: the Indigenous health gap. Int J Epidemiol 2009, 38:470-477.

3. Thomson N, Ride K: Summary of the cardiovascular health status of Indigenous peoples. [accessed 17 June 2011]; [http://www.healthinfonet. ecu.edu.au/chronic-conditions/cvd/reviews/our-review.]

4. Hayman NE, White NE, Spurling GK: Improving Indigenous patients' access to mainstream health services: the Inala experience. Med J Aust 2009, 190:604-606.

5. Turrell G, Kavanagh A, Draper G, Subramanian SV: Do places affect the probability of death in Australia? A multilevel study of area-level disadvantage, individual-level socioeconomic position and all-cause mortality, 1998-2000. J Epidemiol Community Health 2007, 61:13-19.

6. Hill K, Barker B, Vos T: Excess indigenous mortality: are indigenous Australians more severely disadvantaged than other Indigenous populations? Int J Epidemiol 2007, 36:580-589.

7. Mathur S, Moon L, Leigh S: Aboriginal and Torres Strait Islander people with coronary heart disease: further perspectives on health status and treatment. Canberra, Australia: Australian Institute of Health and Welfare; 2006.

8. Katzenellenbogen JM, Sanfilippo FM, Hobbs MST, Briffa TG, Ridout SC, Knuiman MW, Dimer L, Taylor KP, Thompson PL, Thompson SC: Incidence of and case fatality following acute myocardial infarction in Aboriginal and non-Aboriginal Western Australians (2000-2004): a linked data study. Heart Lung Circ 2010, 19:717-725 
9. Brown A: Acute coronary syndromes in Indigenous Australians: opportunities for improving outcomes across the continuum of care. Heart Lung Circ 2010, 19:325-336.

10. Coory MD, Walsh WF: Rates of percutaneous coronary interventions and bypass surgery after acute myocardial infarction in Indigenous patients. Med J Aust 2005, 182:507-512.

11. Bradshaw PJ, Alfonso HS, Finn J, Owen J, Thompson PL: The use of coronary revascularisation procedures in urban Australian Aboriginals and a matched general population: coronary procedures in Aboriginals. Heart Lung Circ 2010, 19:247-250.

12. National Centre for Classification in Health: International Statistical Classification of Diseases and Related Health Problems, 10th Revision, Australian Modification (ICD-10-AM), Australian Classification of Health Interventions (ACHI) and Australian Coding Standards (ACS). 5th edition. Sydney: National Centre for Classification in Health; 2006.

13. Centre for Health Record Linkage [http://www.cherel.org.au]

14. Australian Bureau of Statistics: 3238.0.55.001 - Experimental Estimates of Aboriginal and Torres Strait Islander Australians, Jun 2006. Canberra, Australia: ABS; 2008 [accessed September 17 2010]; [http://www.abs.gov.au/ausstats/ abs@.nsf/Latestproducts/3238.0.55.001Main\%20Features1Jun\%202006? opendocument\&tabname $=$ Summary\&prodno $=3238.0 .55 .001 \&$ issue $=J$ un $\%$ 202006\&num $=\&$ view $=]$

15. Population Health Division: The health of the people of New South Wales Report of the Chief Health Officer. Sydney. NSW Department of Health; 2006 [accessed 7 April 2011]; [http://www.health.nsw.gov.au/publichealth/ chorep/rur/rur_pop_aria_atsi.asp]

16. Australian Bureau of Statistics: 4705.0 Population distribution, Aboriginal and Torres Strait Islander Australians. Canberra, Australia: ABS; 2006 [accessed 31 March 2011]; [http://www.ausstats.abs.gov.au/ausstats/subscriber.nsf/0/ 377284127F903297CA25733700241AC0/\$File/47050_2006.pdf]

17. Australian Bureau of Statistics. 3238.0 - Experimental Estimates and Projections, Aboriginal and Torres Strait Islander Australians, 1991 to 2021. Canberra, Australia: ABS; 2009 [accessed 14 March 2012]; [http://www.abs. gov.au/ausstats/abs@.nsf/0/DDAB99776D7ABDC7CA25762A001CC066? opendocument]

18. Australian Bureau of Statistics. 3235.0 - Population by age and sex, Australia, 2006. Canberra, Australia: ABS; 2007 [accessed 14 March 2012]; [http://www. abs.gov.au/ausstats/abs@.nsf/Products/3235.0 2006 Main+Features New +South+Wales?OpenDocument]

19. Australian Institute of Health and Welfare: Indigenous identification in hospital separations data - quality report. Canberra, Australia: Australian Institute of Health and Welfare; 2010.

20. Australian Institute of Health and Welfare: Improving the quality of Indigenous identification in hospital separations data. Canberra, Australia: Australian Institute of Health and Welfare; 2005.

21. Vermeulen MJ, Tu JV, Schull MJ: ICD-10 adaptations of the Ontario acute myocardial infarction mortality prediction rules performed as well as the original versions. J Clin Epidemiol 2007, 60:971-974.

22. Sundararajan V, Henderson T, Perry C, Muggivan A, Quan H, Ghali WA: New ICD-10 version of the Charlson comorbidity index predicted in-hospital mortality. J Clin Epidemiol 2004, 57:1288-1294.

23. Leyland $\mathrm{AH}$, Groenewegen PP: Multilevel modelling and public health policy. Scand J Public Health 2003, 31:267-274

24. Merlo J, Chaix B, Ohlsson H, Beckman A, Johnell K, Hjerpe P, Råstam L, Larsen $\mathrm{K}$ : A brief conceptual tutorial of multilevel analysis in social epidemiology: using measures of clustering in multilevel logistic regression to investigate contextual phenomena. J Epidemiol Community Health 2006, 60:290-297.

25. SAS Institute Inc: SAS Version 9.1.3 [software]. Cary, North Carolina: SAS; 2003.

26. Rasbash J, Browne WJ, Healy M, Cameron B, Charlton C: MLwiN Version 2.22 [software]. Bristol: Centre for Multilevel Modelling, University of Bristol; 2010.

27. Katzenellenbogen JM, Sanfilippo FM, Hobbs MST, Briffa TG, Ridout SC, Knuiman MW, Dimer L, Taylor KP, Thompson PL, Thompson SC: Aboriginal to non-Aboriginal differentials in 2-year outcomes following non-fatal first-ever acute MI persist after adjustment for comorbidity. Eur J Cardiovasc Prev Rehabil [published online ahead of print 28 July 2011].

28. Brown A: Bridging the survival gap between Indigenous and nonIndigenous Australians: priorities for the road ahead. Heart Lung Circ 2009, 18:96-100

29. Ross JS, Normand SLT, Wang Y, Ko DT, Chen J, Drye EE, Keenan PS, Lichtman JH, Bueno H, Schreiner GC, Krumholz HM: Hospital volume and 30-day mortality for three common medical conditions. N Engl J Med 2010, 362:1110-1118.

30. Alter DA, Naylor CD, Austin PC, Tu JV: Long-term Ml outcomes at hospitals with or without on-site revascularization. JAMA 2001, 285:2101-2108.

31. Wood L, France K, Hunt K, Eades S, Slack-Smith L: Indigenous women and smoking during pregnancy: knowledge, cultural contexts and barriers to cessation. Soc Sci Med 2008, 66:2378-2389.

32. Mezuk B, Rafferty JA, Kershaw KN, Hudson D, Abdou CM, Lee H, Eaton WW Jackson JS: Reconsidering the role of social disadvantage in physical and mental health: stressful life events, health behaviors, race, and depression. Am J Epidemiol 2010, 172:1238-1249.

33. Lawrence M, Dodd Z, Mohor S, Dunn S, de Crespigny C, Power C, MacKean $\mathrm{L}$ : Improving the Patient Journey: Achieving Positive Outcomes for Remote Aboriginal Cardiac Patients. Darwin, Australia: Cooperative Research Centre for Aboriginal Health, Darwin; 2009.

doi:10.1186/1471-2458-12-281

Cite this article as: Randall et al: Mortality after admission for acute myocardial infarction in Aboriginal and non-Aboriginal people in New South Wales, Australia: a multilevel data linkage study. BMC Public Health 2012 12:281

\section{Submit your next manuscript to BioMed Central and take full advantage of:}

- Convenient online submission

- Thorough peer review

- No space constraints or color figure charges

- Immediate publication on acceptance

- Inclusion in PubMed, CAS, Scopus and Google Scholar

- Research which is freely available for redistribution

Submit your manuscript at www.biomedcentral.com/submit
C) BioMed Central 